\title{
Editorial II
}

\section{OUR ROLE IN “ENHANCING RECOVERY” FOLLOWING MAJOR SURGERY}

Major surgery causes a stress response which gives rise to profound physiological, endocrine and metabolic changes. The frequent sequelae are pain, paralytic ileus, increased cardiac demands, impaired pulmonary function and insulin resistance. These changes lead to significant postoperative complications, prolonged hospital stay and increased costs for both the patient and the state.

Enhanced recovery programmes were pioneered in the 1990's by Professor Henrik Kehlet in Denmark and encompasses a multi modal package of techniques aiming to decrease post surgical organ dysfunction and complications, thereby improving post operative recovery ${ }^{1}$.

Pre operative assessment in order to identify co-morbidities and stabilize them and to optimize organ function to face the stress response is a mandatory requisite on our part. This time can be used to educate the patient on the planned perioperative care pathway which is proven to reduce anxiety, analgesic requirements and length of hospital stay. We should consider use of appropriate pre medication in order to reduce the stress response. There is increasing interest in use of $\beta$ blockers ${ }^{2}$ and $\alpha_{2}$ agonists as premedicants. They prevent perioperative cardiovascular morbidity and have analgesic sparing properties.

There is evidence that avoidance of preoperative dehydration reduce postoperative pain and nausea. Prescribing clear fluids to be taken up to $2 \mathrm{hrs}$ prior to surgery has no effect on gastric volume and $\mathrm{pH}$ and on aspiration risk. This is easy for us to practice. Studies have shown that administering clear carbohydrate drinks $800 \mathrm{ml}$ the previous night and $400 \mathrm{ml} 2$ hours prior to surgery reduces insulin resistance and muscle catabolism ${ }^{3}$.
To "Enhance Recovery" our anaesthetic technique should allow rapid recovery, absence of post operative nausea and vomiting and hypothermia and perfect analgesia allowing early mobilization. Our choice to allow a rapid recovery would be to use oxygen/air and isoflurane. The developed world would favour total i.v. anaesthesia (TIVA) with propofol. Analgesia could be provided by a thoracic epidural. This would in addition better preserve exercise capacity, reduce time to ambulation, reduce post operative pulmonary complications and reduce ileus $^{3}$. We need to match the level of epidural placement to the dermatomal level of the incision, use continuous infusions of low concentration bupivacaine and fentanyl and ensure a well working, well secured epidural which would last at least for $48 \mathrm{hrs}$ postoperatively. Multimodal strategies should be used to prevent post operative nausea and vomiting. These would include adequate hydration, minimal opioid use, avoidance of volatile anaesthetics and nitrous oxide and administration of appropriate antiemetics. These strategies are all within are province. Intraoperative hypothermia exacerbates the stress response, increases wound infection, blood loss and coronary events, which would all add to a poor outcome. It is important that we monitor core temperature during major surgery and use forced air warming devices and warm i.v. fluids and blood to prevent hypothermia.

The ideal intraoperative fluid management is a subject of much debate between 'liberal' and 'restrictive' options. Excess fluids impair tissue oxygenation and wound healing, restriction reduce effective circulating volume and organ hypoperfusion. Best approach would be individually tailored 'goal-directed' therapy with tissue perfusion and cellular oxygenation as the goal. The current evidence is for restricted balanced salt crystalloid 
therapy with colloid boluses to achieve goals ${ }^{4}$. Our goals would be mainly clinical, sometimes the central venous pressure; hopefully in the future non invasive stroke volume estimation by oesophageal Doppler or pulse contour analysis and assessment of tissue oxygenation with $\mathrm{ScvO}_{2}$ and lactate levels.

Post operative management includes good analgesia, early enteral nutrition and early mobilization. A multi modal, balance approach for analgesia would minimize opioid use, their side effects allowing for early feeding and mobilization. Evidence shows that early oral intake is safe even after bowel resection ${ }^{5}$. Feeding can be started with small volumes of clear nutritious fluid in $6 \mathrm{hrs}$ post surgery and gradually stepped up to provide required amounts and the change to solid food. Programmed ambulation needs to be started in order to improve gut motility and prevent respiratory and thrombotic complications.

Thus, it can be seen that we have a key role to play in 'Enhanced recovery' programmes to ensure less complications and good post surgical outcomes. Many of the interventions are evidence based but there are still areas of uncertainty. We need to audit our practices in order to contribute to effective "Enhanced recovery".

\section{References}

1. Kehlet H, Wilmore DW. Multi modal strategies to improve surgical outcome, Am. J Surg 2002;183;630-641

2. Devereaux PJ, Beattie WS, Choi PT-L et al. How strong is the evidence for the use of perioperative beta-blockers in patients undergoing noncardiac surgery? A systematic review and metanalysis. BMJ.2005;331;312321

3. Smith CM, Brennan TJ. Thoracic epidural analgesia and acute pain management. Anaesthesiology V 155 No.1; 181- 188

4. Bundgaard-Nielsen M, Secher NH, Kehlet H. 'Liberal' vs. 'restrictive' perioperative fluid therapy--a critical assessment of the evidence. Acta Anaesthesiol Scand 2009;53;843-851

5. Anderson H K, Lewis S J, Thomas S. Early enteral nutrition within 24 hours of colorectal surgery versus later commencement of feeding for postoperative complications Cochrane Database Syst Rev. 2006;(4):CD004080.

\section{Anuja Abayadeera*}

Senior Lecturer in Anaesthesiology

Faculty of Medicine, University of Colombo

*Corresponding author. E-mail: anujaa@sltnet.lk 